\title{
A fitted numerical method for singularly perturbed parabolic reaction-diffusion problems
}

\author{
Justin B. Munyakazi and Kailash C. Patidar
}

\begin{abstract}
This paper treats a time-dependent singularly perturbed reaction-diffusion problem. We semidiscretize the problem in time by means of the classical backward Euler method. We develop a fitted operator finite difference method (FOFDM) to solve the resulting set of linear problems (one at each time level). We prove that the underlying fitted operator satisfies the maximum principle. This result is then used in the error analysis of the FOFDM. The method is shown to be first order convergent in time and second order convergent in space, uniformly with respect to the perturbation parameter. We test the method on several numerical examples to confirm our theoretical findings.
\end{abstract}

\section{Introduction}

When solving numerically time-dependent singularly perturbed problems, it is customary to consider dimension splitting: The problems are semidiscretized in time (for example by using the classical backward Euler or Crank-Nicolson scheme). Then, at each time level, a set of stationary problems is solved using a suitably designed numerical method. Singularly perturbed problems involve a (perturbation) parameter which multiplies the highest derivative term in the model-equation of the problem. The solution to such problems is characterized by layer regions which are narrow parts of the domain over which the solution undergoes abrupt changes. It is well known that classical methods are not appropriate when the perturbation parameter becomes small unless very fine meshes are used for spatial discretization. However, this approach has two side effects: it increases the round-off error and the computational cost. There is a vast literature about non-classical numerical methods. In the context of finite differences, we can group these methods into two classes: the class of fitted mesh methods and the class of fitted operator methods. Both these types of methods have been used to solve stationary singularly perturbed problems in one and several dimensions. As examples, see Linß and Stynes (1999), Lubuma and Patidar (2006), Miller et al. (1996), Munyakazi and Patidar (2010a,b, 2012), Patidar (2005, 2007), Roos et al. (1996), Shishkin (1986, 2005). It should be noted that the discovery/development of fitted mesh methods is anterior to that of the fitted operator ones. The analysis of the latter is simpler due to the fact that they are based on uniform meshes unlike the former where non-uniform meshes are designed. 
This paper is concerned with the problem of finding a numerical solution of the following timedependent singularly perturbed reaction-diffusion equation

$$
u_{t}+L_{x, \varepsilon} u=f(x, t), \quad(x, t) \in Q=\Omega \times(0, T] \equiv(0,1) \times(0, T),
$$

subject to the initial and boundary conditions

$$
u(x, 0)=0, \quad x \in \bar{\Omega}, \quad u(0, t)=u(1, t)=0, t \in(0, T] .
$$

The spatial differential operator is defined as

$$
L_{x, \varepsilon} u \equiv-\varepsilon u_{x x}+b(x, t) u .
$$

where $\varepsilon>0, b \geq \beta>0$, for all $(x, t) \in \bar{Q}$ and the data $b, f \in C^{(4,2)}(\bar{Q})$. Also, we impose the following compatibility conditions between data of problem (1), (2) so that the exact solution $u(x, t) \in C(4,2)(\bar{Q})$ :

$$
f(0,0)=f(1,0)=0, \quad\left(L_{x, \varepsilon} f\right)(0,0)=f_{t}(0,0), \quad\left(L_{x, \varepsilon} f\right)(1,0)=f_{t}(1,0) .
$$

The solution of problem (1), (2) is such that Hemker et al. (2000)

$$
\left|u^{(k, m)}(x, t)\right| \leq C\left[1+\varepsilon^{-k / 2}(\exp (-\sqrt{\beta / \varepsilon} x)+\exp (-\sqrt{\beta / \varepsilon}(1-x))],\right.
$$

where $0 \leq k+2 m \leq 4$. Bounds (4) highlight the fact that the solution of (1), (2) has a boundary layer at $x=0$ and $x=1$.

Many researchers have treated time-dependent singularly perturbed problems. Below, we mention a few examples. A uniformly convergent scheme for convection-diffusion parabolic problems is developed on a nonuniform mesh in Clavero et al. (2003). Numerical methods are designed in Jorge and Bujanda (2004) to integrate reaction-diffusion parabolic problems with non-linear reaction terms. An alternating direction method applied to singularly perturbed reaction-diffusion problems is analyzed in $\operatorname{Lin} \beta$ and Madden (2010). In Clavero and Gracia (2010), a uniformly convergent finite difference method is designed for parabolic reactiondiffusion problems in one dimension. A Schwarz domain decomposition method is designed in Rao and Kumar (2011) for a system of coupled singularly perturbed reaction-diffusion equations.

All the above methods are applied on nonuniform meshes (of Shishkin, Bakhvalov or Vulanovic types). Inspired by the simplicity of analysis on uniform meshes and having

\section{http://repository.uwc.ac.za}


noticed that, up to the best of our knowledge, no fitted operator finite difference methods developed for problems of the type (1), (2), our aim in this paper is to design one such method. Also, we prove that the method is second order uniformly convergent with respect to the singular perturbation parameter. We test the method on several numerical examples to confirm our theoretical findings.

The remainder of this paper is organized as follows: in Sect. 2, we discretize problem (1), (2) in time by using the classical backward Euler scheme. This results in a set of linear reaction-diffusion problems (one at each time-level). These problems are discretized in Sect. 3. A result on the stability of this method is proved in this section as well as the discrete maximum principle satisfied by the underlying discrete operator. Section 4 is devoted to the analysis of the fully discrete method presented in Sect. 3. Through this analysis, we show that the method is uniformly convergent with respect to $\varepsilon$. Several numerical examples are provided in Sect. 5 to confirm in practice the theoretical findings. Some concluding remarks and future plans end the paper.

\section{The time semidiscretization}

In this paper, we first discretize the problem in time by means of the backward Euler method. This results in a linear problem in space at each time level which we discretize using a fitted operator method in next section. The time interval $[0, T]$ follows:

$$
\bar{\omega}^{K}=\left\{t_{k}=k \tau, \quad 0 \leq k \leq K, \quad \tau=T / K\right\} .
$$

Then the problem (1), (2) is discretized on $\bar{\omega} K$ in the following manner:

$$
\begin{gathered}
\frac{z\left(x, t_{k}\right)-z\left(x, t_{k-1}\right)}{\tau}+L_{x, \varepsilon}\left(z\left(x, t_{k}\right)\right)=f\left(x, t_{k}\right), \quad 1 \leq k \leq K, \\
z(x, 0)=0, \text { for all } x \in(0,1), \quad z\left(0, t_{k}\right)=z\left(1, t_{k}\right)=0 .
\end{gathered}
$$

We now introduce auxiliary problems in order to define the local error. We have

$$
\frac{\hat{z}\left(x, t_{k}\right)-u\left(x, t_{k-1}\right)}{\tau}+L_{x, \varepsilon}\left(\hat{z}\left(x, t_{k}\right)\right)=f\left(x, t_{k}\right), \quad 1 \leq k \leq K, \quad z\left(0, t_{k}\right)=z\left(1, t_{k}\right)=0 .(8)
$$

We rewrite Eq. (8) as

$$
\left(I+\tau L_{x, \varepsilon}\right) \hat{z}\left(x, t_{k}\right)=\tau f\left(x, t_{k}\right)+u\left(x, t_{k-1}\right) .
$$

Subtracting Eq. (9) from (1) and expanding $u(x, t k-1)$ in Taylor series we obtain 


$$
L_{x, \varepsilon} E_{k}=-\frac{E_{k}}{\tau}+\frac{\tau}{2} u_{t t}(x, t)+\text { higher order terms }
$$

where $E_{k}=u\left(x, t_{k}\right)-\hat{z}\left(x, t_{k}\right)$ is the local error associated with method (6) and $u t t$ is the second derivative of $u$ with respect to time. Since $\|\left(I+\tau L_{x, \varepsilon}\right)^{-1}|| \bar{Q} \leq 1 /(1+\tau \beta)$ and in virtue of (4), we see that Lemma 2.1 (Clavero et al. (2000)) The local error associated with method (6) satisfies

$$
\left\|E_{k}\right\|_{\Omega} \leq C \tau^{2}, \quad 1 \leq k \leq K
$$

The result on the global error follows naturally.

Theorem 2.2 (Clavero et al. (2000)) The global error associated with method (6), defined by $e k=u\left(x, t_{k}\right)-z\left(x, t_{k}\right)$, satisfies,

$$
\left\|e_{k}\right\|_{\bar{\Omega}} \leq C \tau, \quad 1 \leq k \leq K
$$

and therefore the backward Euler method is a first order uniformly convergent scheme.

The next Lemma gives the bounds of the solution of the semidiscrete problem (6) as well as those of its derivatives.

Lemma 2.3 Let $z(x, t k)$ be the solution of problem (6) at the time level $k$. Then, we have

$$
\left|z^{(m)}\left(x, t_{k}\right)\right| \leq C\left[1+\varepsilon^{-m / 2}(\exp (-\sqrt{\beta / \varepsilon} x)+\exp (-\sqrt{\beta / \varepsilon}(1-x))]\right.
$$

where $0 \leq m \leq 4$ and $1 \leq k \leq K$.

Proof See Clavero and Gracia (2010) At each time level, a boundary value problem is obtained by using the backward Euler method. In the next section we design a fitted operator finite difference (FOFDM) to solve this set of linear reaction-diffusion problems.

\section{The fully discrete method}

Let $N$ be a positive integer. We consider the following partition of the interval $[0,1]$ which we denote by $\bar{\Omega}^{N}$ :

$x_{0}=0, \quad x_{i}=x_{0}+i h, \quad i=1(1) n, \quad h=x_{i}-x_{i-1}, \quad x_{N}=1$

and let $\bar{Q} N, K=\bar{\Omega}^{N} \times \omega^{K}$ be the grid for the $x, t$-variables, and $Q^{N, K}=\bar{Q}^{N, K} \cap Q$. 
In the rest of this paper, we adopt the notation $w_{j}^{k}=w\left(x_{j}, t_{k}\right)$ and denote the approximations of the $z_{j}^{k}$. at the grid points $\left(x_{j}, t_{k}\right)$ by the unknowns $U_{j}^{k}$.

Using the theory of difference equations for problems in one dimension Mickens (1994), we construct the following scheme:

$$
L_{\varepsilon}^{N, K} U_{j}^{k} \equiv \frac{U_{j}^{k}-U_{j}^{k-1}}{\tau}+L_{x, \varepsilon}^{N} U_{j}^{k}=f_{j}^{k},
$$

where

$$
L_{x, \varepsilon}^{N} U_{j}^{k} \equiv-\varepsilon\left[\frac{U_{j+1}^{k}-2 U_{j}^{k}+U_{j-1}^{k}}{\phi_{j}^{2}}\right]+b_{j}^{k} U_{j}^{k}
$$

with the discrete initial and boundary conditions

$$
\begin{aligned}
U_{j}^{0} & =0, \quad j=0, \ldots, N, \\
U_{0}^{k} & =U_{N}^{k}=0 \quad 1 \leq k \leq K,
\end{aligned}
$$

where

$$
\phi_{j}=\frac{2}{\rho_{j}} \sinh \left(\frac{\rho_{j} h}{2}\right) \text { and } \rho_{j}=\sqrt{\left(\frac{1}{\tau}+b_{j}^{k}\right) \varepsilon^{-1}} .
$$

The scheme (10)-(17) is a tridiagonal system of linear equations

$$
A U=F
$$

where the corresponding entries of $A$ and $F$ are

$$
\begin{aligned}
A_{i j}=r_{j}^{-}, \quad i & =j+1 ; j=1,2, \ldots, n-2, \\
A_{i j}=r_{j}^{c}, \quad i & =j ; j=1,2, \ldots, n-1, \\
A_{i j}=r_{j}^{+}, \quad i & =j-1 ; j=2,3, \ldots, n-1, \\
F_{j} & =f_{j}^{k}+U_{j}^{k-1} / \tau, j=1,2, \ldots, n-1,
\end{aligned}
$$

With

$$
r_{j}^{-}=-\frac{\varepsilon}{\phi_{j}^{2}}, \quad r_{j}^{c}=\frac{2 \varepsilon}{\phi_{j}^{2}}+\frac{1}{\tau}+b_{j}^{k} \quad \text { and } \quad r_{j}^{+}=-\frac{\varepsilon}{\phi_{j}^{2}} .
$$

We refer to the method developed above as the FOFDM for the parabolic problem (1), (2). 
In the discussion below, $M$ may denotes a positive constant which may take different values in different equations and inequalities but is always independent of $\varepsilon$ and the step-sizes $h$ and $\tau$.

Before we embark on the analysis of the scheme developed above, we will present a number of results in the form of Lemmas which play a crucial role in the said analysis.

Lemma 3.1 [Discrete maximum principle] Let $L_{\varepsilon}^{N, K}$ be the discrete operator given in (10) and $\Psi_{j}^{k}$ any mesh function satisfying $\Psi_{j}^{0} \geq 0, \quad 0 \leq j \leq N, \Psi_{0}^{k} \geq 0, \quad \Psi_{N}^{k} \geq 0, \quad 1 \leq k \leq K$ and $L_{\varepsilon}^{N, K} \Psi_{j}^{k} \geq 0$ in $Q^{N, K}$. Then $\Psi_{j}^{k} \geq 0$ in $\bar{Q}^{N, K}$.

Proof Let $(s, l)$ be indices such that

$$
\Psi_{s}^{l}=\min _{(j, k)} \Psi_{j}^{k}, \quad \Psi_{j}^{k} \in \bar{Q}^{N, K} .
$$

Assume that $\Psi_{s}^{l}<0$. It is clear that $(s, l) \in\{1,2, \ldots, N-1\} \times\{1,2, \ldots, K-1\}$ otherwise $\Psi_{s}^{l} \geq 0$. We observe that $\Psi_{s+1}^{l}-\Psi_{s}^{l}>0$ and $\Psi_{s-1}^{l}-\Psi_{s}^{l}>0$. Therefore

$$
L_{\varepsilon}^{N, K} \Psi_{s}^{l}<0
$$

which is a contradiction. 
Lemma 3.2 [Uniform stability estimate] At any time level $k$, if $Z_{j}^{k}$ is any mesh function such that $Z_{0}^{k}=Z_{N}^{k}=0$, then

$$
\left|Z_{i}^{k}\right| \leq \frac{1}{\beta} \max _{1 \leq j \leq N-1}\left|L_{\varepsilon}^{N, K} Z_{j}^{k}\right|, \quad \text { for } 0<i<N .
$$

Proof Let

$$
P=\frac{1}{\beta} \max _{1 \leq j \leq N-1}\left|L_{\varepsilon}^{N, K} Z_{j}^{k}\right|
$$

and $\left(\Psi^{ \pm}\right)_{j}^{k}$ be the mesh function defined by

$$
\left(\Psi^{ \pm}\right)_{j}^{k}=P \pm Z_{j}^{k}
$$

It is clear that $\left(\Psi^{ \pm}\right)_{0}^{k}=\left(\Psi^{ \pm}\right)_{N}^{k}=P>0$. Also, for $1 \leq j \leq N-1$, we have

$$
\begin{aligned}
L_{\varepsilon}^{N, K}\left(\Psi^{ \pm}\right)_{j}^{k}= & -\varepsilon\left[\frac{P \pm Z_{j+1}^{k}-2\left(P \pm Z_{j}^{k}\right)+P \pm Z_{j-1}^{k}}{\phi_{j}^{2}}\right] \\
& +\left(\frac{1}{\tau}+b_{j}^{k}\right)\left(P \pm Z_{j}^{k}\right) \\
= & \left(\frac{1}{\tau}+b_{j}^{k}\right) P \pm L_{\varepsilon}^{N, K} Z_{j}^{k} \\
= & \frac{1}{\beta}\left(\frac{1}{\tau}+b_{j}^{k}\right) \max \left|L_{\varepsilon}^{N, K} Z_{j}^{k}\right| \pm L_{\varepsilon}^{N, K} Z_{j}^{k} .
\end{aligned}
$$

Since $1 / \tau+b_{j}^{k}>\beta$, we have $L_{\varepsilon}^{N, K}\left(\Psi^{ \pm}\right)_{j}^{k} \geq 0$. Thus, by the discrete maximum principle (Lemma 3.1), we obtain

$$
\left(\Psi^{ \pm}\right)_{j}^{k} \geq 0 \text { for } 0 \leq j \leq N
$$

We are now ready to analyze the method.

\section{Error analysis of the fully discrete method}

For the sake of simplicity, we will ignore the time level index. The local truncation error of our FOFDM is obtained from (10) in the following manner: 


$$
\begin{aligned}
L_{\varepsilon}^{N, K}\left(U_{j}-z_{j}\right) & =\left(L_{x, \varepsilon}-L_{x, \varepsilon}^{N}\right) z_{j} \\
& =-\varepsilon z_{j}^{\prime \prime}+\left[\varepsilon \frac{z_{j+1}-2 z_{j}+z_{j-1}}{\phi_{j}^{2}}\right] \\
& =-\varepsilon z_{j}^{\prime \prime}+\frac{\varepsilon}{\phi_{j}^{2}}\left[h^{2} z_{j}^{\prime \prime}+\frac{h^{4}}{12} z^{(i v)}\left(\xi_{j}\right)\right], \quad \xi_{j} \in\left(x_{j-1}, x_{j+1}\right)
\end{aligned}
$$

Note that we have used Taylor expansions of $z_{j+1}$ and $z_{j-1}$. A truncated Taylor expansion of $1 / \phi_{j}^{2}$ of order five reads

$$
\frac{1}{\phi_{j}^{2}}=\frac{\rho_{j}^{2}}{4}\left(\frac{4}{\rho_{j}^{2} h^{2}}-\frac{1}{3}+\frac{\rho_{j}^{2} h^{2}}{60}\right) .
$$

Now, using (17) in (16), we obtain

$$
\begin{aligned}
L_{\varepsilon}^{N, K}\left(U_{j}-z_{j}\right)= & {\left[\frac{\varepsilon}{12}\left(z^{(i v)}\left(\xi_{j}\right)-\rho_{j}^{2} z_{j}^{\prime \prime}\right)\right] h^{2} } \\
& +\left[\varepsilon \rho_{j}^{2}\left(\frac{\rho_{j}^{2}}{240} z_{j}^{\prime \prime}-\frac{1}{144} z^{(i v)}\left(\xi_{j}\right)\right)\right] h^{4}+\frac{\varepsilon \rho_{j}^{4}}{2880} z^{(i v)}\left(\xi_{j}\right) h^{6}
\end{aligned}
$$

The following Lemma will be useful in the continuation of this discussion.

Lemma 4.1 For a fixed mesh and for all integers $m$, we have

$$
\begin{aligned}
& \lim _{\varepsilon \rightarrow 0} \max _{1 \leq j \leq N-1} \frac{\exp \left(-M x_{j} / \sqrt{\varepsilon}\right)}{\varepsilon^{m / 2}}=0 \text { and } \\
& \lim _{\varepsilon \rightarrow 0} \max _{1 \leq j \leq N-1} \frac{\exp \left(-M\left(1-x_{j}\right) / \sqrt{\varepsilon}\right)}{\varepsilon^{m / 2}}=0 .
\end{aligned}
$$

Proof See Munyakazi and Patidar (2010a) or Patidar (2007)

Using Lemmas 2.3 and 4.1, we obtain

$$
\left|L_{\varepsilon}^{N, K}\left(U_{j}-z_{j}\right)\right| \leq M h^{2} .
$$

Then by Lemma 3.2 and re-instating the dropped time level index, we have proved the following theorem.

Theorem 4.2 Let ${ }_{j}^{k}$ be the numerical solution of (10) and $z(x, t k)$ the solution of (6) both at the time level $k$. Then and therefore the FOFDM is a second order uniformly convergent scheme in space. 
The global error satisfies

$$
\left|U_{j}^{k}-u_{j}^{k}\right| \leq\left|U_{j}^{k}-z_{j}^{k}\right|+\left|z_{j}^{k}-u_{j}^{k}\right|,
$$

thus, combining Theorems 2.2 and 4.2 leads to the final result of this work:

Theorem 4.3 Let $U_{j}^{k}$ be the numerical solution of (10) and $u(x, t)$ the solution of (1), (2). Then

$$
\max _{0 \leq j \leq N, 1 \leq k \leq K}\left|U_{j}^{k}-u_{j}^{k}\right| \leq M\left(h^{2}+\tau\right) .
$$

\section{Numerical results}

In this section, we illustrate our method with four numerical examples. The exact solutions to our test examples are not known. We therefore use a variant of the double mesh principle to estimate the errors of the computed approximations.

The maximum errors at all mesh points are calculated using the formulas

$$
E^{\varepsilon, N, \tau}=\max _{0 \leq j \leq N ; 0 \leq k \leq K}\left|U_{j, k}^{\varepsilon, N, \tau}-U_{j, k}^{\varepsilon, 2 N, \frac{\tau}{4}}\right|,
$$

Where ${ }^{U_{j, k}^{\varepsilon, h, \tau}}$ is the approximate solution obtained using a constant time step $\tau$ and space step $h$. Likewise, ${ }^{U_{j, k}^{\varepsilon, 2 N, \frac{\tau}{4}}}$ is computed using the constant time step $\tau / 4$ and space step $h / 2$. The numerical rates of convergence are computed using the formula Doolan et al. (1980):

$$
r_{l} \equiv r_{\varepsilon, l}:=\log _{2}\left(E^{\varepsilon, N_{l}, \tau} / E^{\varepsilon, 2 N_{l}, \frac{\tau}{4}}\right), \quad l=1,2, \ldots
$$

Furthermore, we compute

$$
E_{N, \tau}=\max _{0<\varepsilon \leq 1} E^{\varepsilon, N, \tau}
$$

and the numerical rate of uniform convergence as

$$
R_{N, \tau}=\log _{2}\left(E_{N, \tau} / E_{2 N, \tau / 4}\right) .
$$

Example 5.1 Clavero and Gracia (2005) 


$$
\begin{gathered}
u_{t}-\varepsilon u_{x x}+\frac{1+x^{2}}{2} u=t^{3}, \quad(x, t) \in(0,1) \times(0,1] \\
u(x, 0)=0, \quad x \in[0,1] ; \quad u(0, t)=u(1, t)=0, \quad t \in(0,1] .
\end{gathered}
$$

Our theoretical analysis shows that the method developed is second order uniformly convergent in space independently of the perturbation parameter $\varepsilon$ as mentioned in Theorem 4.2. This is confirmed by numerical results presented in Tables 1 and 2 where we computed the maximum errors $E^{\mathcal{E}, N, \tau}$ and the corresponding rates of convergence $r l$, respectively. The last row of Table 1 gives $E_{N, \tau}$ while the rate of uniform convergence $R N, \tau$ is displayed in the last row of Table 2.

The next three examples are chosen to show that the effect of the compatibility conditions is not as severe as it is in the case of numerical methods based on non-uniform meshes.

Table 1 Results for Example 5.1: maximum errors $E^{\varepsilon, N, \tau}$

\begin{tabular}{llllll}
\hline$\varepsilon$ & $N=32$ & $N=64$ & $N=128$ & $N=256$ & $N=512$ \\
& $\tau=0.1$ & $\tau=0.1 / 4$ & $\tau=0.1 / 4^{2}$ & $\begin{array}{l} \\
\tau=0.1 / 4^{3}\end{array}$ & $\begin{array}{l} \\
\tau=0.1 / 4^{4}\end{array}$ \\
\hline $2^{-6}$ & $2.88 \mathrm{E}-02$ & $0.71 \mathrm{E}-02$ & $0.18 \mathrm{E}-02$ & $4.42 \mathrm{E}-04$ & $1.10 \mathrm{E}-04$ \\
$2^{-8}$ & $2.97 \mathrm{E}-02$ & $0.73 \mathrm{E}-02$ & $0.18 \mathrm{E}-02$ & $4.54 \mathrm{E}-04$ & $1.13 \mathrm{E}-04$ \\
$2^{-10}$ & $3.01 \mathrm{E}-02$ & $0.74 \mathrm{E}-02$ & $0.18 \mathrm{E}-02$ & $4.58 \mathrm{E}-04$ & $1.15 \mathrm{E}-04$ \\
$2^{-16}$ & $3.02 \mathrm{E}-02$ & $0.74 \mathrm{E}-02$ & $0.18 \mathrm{E}-02$ & $4.59 \mathrm{E}-04$ & $1.15 \mathrm{E}-04$ \\
$2^{-18}$ & $3.02 \mathrm{E}-02$ & $0.74 \mathrm{E}-02$ & $0.18 \mathrm{E}-02$ & $4.59 \mathrm{E}-04$ & $1.15 \mathrm{E}-04$ \\
$2^{-20}$ & $3.02 \mathrm{E}-02$ & $0.74 \mathrm{E}-02$ & $0.18 \mathrm{E}-02$ & $4.59 \mathrm{E}-04$ & $1.15 \mathrm{E}-04$ \\
$\vdots$ & $\vdots$ & $\vdots$ & $\vdots$ & $\vdots$ & $\vdots$ \\
$2^{-50}$ & $3.02 \mathrm{E}-02$ & $0.74 \mathrm{E}-02$ & $0.18 \mathrm{E}-02$ & $4.59 \mathrm{E}-04$ & $1.15 \mathrm{E}-04$ \\
$E_{N, \tau}$ & $3.02 \mathrm{E}-02$ & $0.74 \mathrm{E}-02$ & $0.18 \mathrm{E}-02$ & $4.59 \mathrm{E}-04$ & $1.15 \mathrm{E}-04$ \\
\hline
\end{tabular}

Table 2 Results for Example 5.1: convergence rates $r_{l}, N_{l}=32 \times 2^{l-1}, \quad l=1(1) 4$

\begin{tabular}{lllll}
\hline$\varepsilon$ & $r_{1}$ & $r_{2}$ & $r_{3}$ & $r_{4}$ \\
\hline $2^{-6}$ & 2.0202 & 1.9798 & 2.0266 & 2.0004 \\
$2^{-8}$ & 2.0245 & 2.0199 & 1.9875 & 2.0004 \\
$2^{-10}$ & 2.0242 & 2.0395 & 1.974 & 2.0004 \\
$2^{-16}$ & 2.029 & 2.0395 & 1.9702 & 2.0005 \\
$2^{-18}$ & 2.029 & 2.0395 & 1.9702 & 2.0005 \\
$2^{-20}$ & 2.029 & 2.0395 & 1.9702 & 2.0005 \\
$\vdots$ & $\vdots$ & $\vdots$ & $\vdots$ & $\vdots$ \\
$2^{-50}$ & 2.029 & 2.0395 & 1.9702 & 2.0005 \\
$R_{N, \tau}$ & 2.029 & 2.0395 & 1.9702 & 2.0005 \\
\hline
\end{tabular}


Example 5.2 Clavero and Gracia (2010)

$$
\begin{gathered}
u_{t}-\varepsilon u_{x x}+\frac{1+x^{2}}{2} u=\exp (x)-1+\sin (\pi x), \quad(x, t) \in(0,1) \times(0,1] \\
u(x, 0)=0, x \in[0,1] ; u(0, t)=u(1, t)=0, t \in(0,1] .
\end{gathered}
$$

Example 5.3 Clavero and Gracia (2005)

$$
\begin{aligned}
& u_{t}-\varepsilon u_{x x}+\frac{1+x^{2}}{2} u=(1+t)^{3}, \quad(x, t) \in(0,1) \times(0,1] \\
& u(x, 0)=0, \quad x \in[0,1] ; \quad u(0, t)=u(1, t)=0, \quad t \in(0,1] .
\end{aligned}
$$

Example 5.4 The following example was also considered:

$$
\begin{gathered}
u_{t}-\varepsilon u_{x x}+\frac{2+x}{3} u=1+t^{2}, \quad(x, t) \in(0,1) \times(0,1] ; \\
u(x, 0)=0, \quad x \in[0,1] ; \quad u(0, t)=u(1, t)=0, \quad t \in(0,1] .
\end{gathered}
$$

For each of these test examples, we computed the maximum errors $E^{\mathcal{E}, N, \tau}$ (see Tables 3,5 and 7) and the corresponding rates of convergence $r l$ (see Tables 4, 6 and 8). The regularity of the exact solution is lowered by the non satisfaction of the compatibility conditions (3). However, this does not impact negatively on the $\mathcal{\varepsilon}$-uniform order of convergence of our method unlike what is observed in Clavero and Gracia (2005) where the third order of uniform convergence is reduced to one. 
Table 3 Results for Example 5.2: maximum errors $E^{\varepsilon, N, \tau}$

\begin{tabular}{llllll}
\hline$\varepsilon$ & $N=32$ & $N=64$ & $N=128$ & $N=256$ & $N=512$ \\
& $\tau=0.1$ & $\tau=0.1 / 4$ & $\begin{array}{l}N=0.1 / 4^{2} \\
\tau=0.1 / 4^{3}\end{array}$ & $\begin{array}{l}N=0.1 / 4^{4} \\
\tau\end{array}$ \\
\hline $2^{-6}$ & $2.74 \mathrm{E}-02$ & $0.69 \mathrm{E}-02$ & $0.17 \mathrm{E}-02$ & $4.34 \mathrm{E}-04$ & $1.09 \mathrm{E}-04$ \\
$2^{-8}$ & $3.43 \mathrm{E}-02$ & $0.87 \mathrm{E}-02$ & $0.22 \mathrm{E}-02$ & $5.44 \mathrm{E}-04$ & $1.36 \mathrm{E}-04$ \\
$2^{-10}$ & $3.83 \mathrm{E}-02$ & $0.97 \mathrm{E}-02$ & $0.24 \mathrm{E}-02$ & $6.11 \mathrm{E}-04$ & $1.53 \mathrm{E}-04$ \\
$2^{-16}$ & $4.13 \mathrm{E}-02$ & $1.05 \mathrm{E}-02$ & $0.27 \mathrm{E}-02$ & $6.66 \mathrm{E}-04$ & $1.67 \mathrm{E}-04$ \\
$2^{-18}$ & $4.13 \mathrm{E}-02$ & $1.05 \mathrm{E}-02$ & $0.27 \mathrm{E}-02$ & $6.66 \mathrm{E}-04$ & $1.67 \mathrm{E}-04$ \\
$2^{-20}$ & $4.13 \mathrm{E}-02$ & $1.05 \mathrm{E}-02$ & $0.27 \mathrm{E}-02$ & $6.66 \mathrm{E}-04$ & $1.67 \mathrm{E}-04$ \\
$\vdots$ & $\vdots$ & $\vdots$ & $\vdots$ & $\vdots$ & $\vdots$ \\
$2^{-50}$ & $4.13 \mathrm{E}-02$ & $1.05 \mathrm{E}-02$ & $0.27 \mathrm{E}-02$ & $6.66 \mathrm{E}-04$ & $1.67 \mathrm{E}-04$ \\
$E_{N, \tau}$ & $4.13 \mathrm{E}-02$ & $1.05 \mathrm{E}-02$ & $0.27 \mathrm{E}-02$ & $6.66 \mathrm{E}-04$ & $1.67 \mathrm{E}-04$ \\
\hline
\end{tabular}

Table 4 Results for

Example 5.2: convergence rates $r_{l}, N_{l}=32 \times 2^{l-1}, \quad l=1(1) 4$

\begin{tabular}{lllll}
\hline$\varepsilon$ & $r_{1}$ & $r_{2}$ & $r_{3}$ & $r_{4}$ \\
\hline $2^{-6}$ & 1.9895 & 2.0211 & 1.9699 & 1.9997 \\
$2^{-8}$ & 1.9791 & 1.9835 & 2.0152 & 1.9997 \\
$2^{-10}$ & 1.9813 & 2.015 & 1.9736 & 1.9996 \\
$2^{-16}$ & 1.9758 & 1.9594 & 2.0191 & 1.9975 \\
$2^{-18}$ & 1.9758 & 1.9594 & 2.0191 & 1.9975 \\
$2^{-20}$ & 1.9758 & 1.9594 & 2.0191 & 1.9975 \\
$\vdots$ & $\vdots$ & $\vdots$ & $\vdots$ & $\vdots$ \\
$2^{-50}$ & 1.9758 & 1.9594 & 2.0191 & 1.9975 \\
$R_{N, \tau}$ & 1.9758 & 1.9594 & 2.0191 & 1.9975 \\
\hline
\end{tabular}

Table 5 Results for Example 5.3: maximum errors $E^{\varepsilon, N, \tau}$

\begin{tabular}{llllll}
\hline$\varepsilon$ & $N=32$ & $N=64$ & $N=128$ & $N=256$ & $N=512$ \\
& $\tau=0.1$ & $\tau=0.1 / 4$ & $\tau=0.1 / 4^{2}$ & $\begin{array}{l}N=0.1 / 4^{3} \\
\tau=0.1 / 4^{4}\end{array}$ \\
\hline $2^{-6}$ & $1.502 \mathrm{E}-01$ & $3.79 \mathrm{E}-02$ & $0.95 \mathrm{E}-02$ & $0.24 \mathrm{E}-02$ & $5.93 \mathrm{E}-04$ \\
$2^{-8}$ & $1.613 \mathrm{E}-01$ & $4.05 \mathrm{E}-02$ & $1.01 \mathrm{E}-02$ & $0.25 \mathrm{E}-02$ & $6.34 \mathrm{E}-04$ \\
$2^{-10}$ & $1.654 \mathrm{E}-01$ & $4.15 \mathrm{E}-02$ & $1.04 \mathrm{E}-02$ & $0.26 \mathrm{E}-02$ & $6.50 \mathrm{E}-04$ \\
$2^{-16}$ & $1.67 \mathrm{E}-01$ & $4.18 \mathrm{E}-02$ & $1.05 \mathrm{E}-02$ & $0.26 \mathrm{E}-02$ & $6.54 \mathrm{E}-04$ \\
$2^{-18}$ & $1.67 \mathrm{E}-01$ & $4.18 \mathrm{E}-02$ & $1.05 \mathrm{E}-02$ & $0.26 \mathrm{E}-02$ & $6.54 \mathrm{E}-04$ \\
$2^{-20}$ & $1.67 \mathrm{E}-01$ & $4.18 \mathrm{E}-02$ & $1.05 \mathrm{E}-02$ & $0.26 \mathrm{E}-02$ & $6.54 \mathrm{E}-04$ \\
$\vdots$ & $\vdots$ & $\vdots$ & $\vdots$ & $\vdots$ & $\vdots$ \\
$2^{-50}$ & $1.67 \mathrm{E}-01$ & $4.18 \mathrm{E}-02$ & $1.05 \mathrm{E}-02$ & $0.26 \mathrm{E}-02$ & $6.54 \mathrm{E}-04$ \\
$E_{N, \tau}$ & $1.67 \mathrm{E}-01$ & $4.18 \mathrm{E}-02$ & $1.05 \mathrm{E}-02$ & $0.26 \mathrm{E}-02$ & $6.54 \mathrm{E}-04$ \\
\hline
\end{tabular}

\section{Concluding remarks and future plans}

A time-dependent singularly perturbed reaction-diffusion problem in one dimension was treated. We semidiscretized the problem by means of the classical backward Euler method. The resulting set of linear reaction-diffusion problems was then solved by a novel fitted operator finite difference method. The whole process was shown to be first order uniformly convergent in time and second order uniformly convergent in space with respect to the perturbation parameter. Numerical results presented in Tables 1, 2, 3, 4, 5, 6, 7 and 8 confirm the theoretical estimates given in Theorem 4.2. Moreover, we noticed that the compatibility 
conditions imposed between the data of the problem could be relaxed without impacting negatively on the performance of the method proposed in this paper.

We are currently investigating similar techniques for time-dependent singularly perturbed convection-diffusion problems. 
Table 6 Results for Example 5.3: convergence rates $r_{l}, N_{l}=32 \times 2^{l-1}, \quad l=1(1) 4$

\begin{tabular}{lllll}
\hline$\varepsilon$ & $r_{1}$ & $r_{2}$ & $r_{3}$ & $r_{4}$ \\
\hline $2^{-6}$ & 1.9866 & 1.9962 & 1.9849 & 2.0162 \\
$2^{-8}$ & 1.9938 & 2.0036 & 2.0144 & 1.979 \\
$2^{-10}$ & 1.9948 & 1.9965 & 2.0000 & 2.001 \\
$2^{-16}$ & 1.9983 & 1.9931 & 2.0138 & 1.9911 \\
$2^{-18}$ & 1.9983 & 1.9931 & 2.0138 & 1.9911 \\
$2^{-20}$ & 1.9983 & 1.9931 & 2.0138 & 1.9911 \\
$\vdots$ & $\vdots$ & $\vdots$ & $\vdots$ & $\vdots$ \\
$2^{-50}$ & 1.9983 & 1.9931 & 2.0138 & 1.9911 \\
$R_{N, \tau}$ & 1.9983 & 1.9931 & 2.0138 & 1.9911 \\
\hline
\end{tabular}

Table 7 Results for Example 5.4: maximum errors $E^{\varepsilon, N, \tau}$

\begin{tabular}{llllll}
\hline$\varepsilon$ & $N=32$ & $N=64$ & $N=128$ & $N=256$ & $\begin{array}{l}N=512 \\
\tau=0.1 / 4^{4}\end{array}$ \\
\hline $2^{-6}$ & $0.93 \mathrm{E}-02$ & $0.27 \mathrm{E}-02$ & $7.06 \mathrm{E}-04$ & $1.79 \mathrm{E}-04$ & $4.48 \mathrm{E}-05$ \\
$2^{-8}$ & $1.13 \mathrm{E}-02$ & $0.34 \mathrm{E}-02$ & $9.01 \mathrm{E}-04$ & $2.29 \mathrm{E}-04$ & $5.73 \mathrm{E}-05$ \\
$2^{-10}$ & $1.95 \mathrm{E}-02$ & $0.90 \mathrm{E}-02$ & $0.24 \mathrm{E}-02$ & $6.37 \mathrm{E}-04$ & $1.62 \mathrm{E}-04$ \\
$2^{-16}$ & $1.18 \mathrm{E}-02$ & $0.29 \mathrm{E}-02$ & $7.18 \mathrm{E}-04$ & $1.80 \mathrm{E}-04$ & $4.49 \mathrm{E}-05$ \\
$2^{-18}$ & $1.18 \mathrm{E}-02$ & $0.29 \mathrm{E}-02$ & $7.18 \mathrm{E}-04$ & $1.80 \mathrm{E}-04$ & $4.49 \mathrm{E}-05$ \\
$2^{-20}$ & $1.18 \mathrm{E}-02$ & $0.29 \mathrm{E}-02$ & $7.18 \mathrm{E}-04$ & $1.80 \mathrm{E}-04$ & $4.49 \mathrm{E}-05$ \\
$\vdots$ & $\vdots$ & $\vdots$ & $\vdots$ & $\vdots$ & $\vdots$ \\
$2^{-50}$ & $\vdots$ & $0.29 \mathrm{E}-02$ & $7.18 \mathrm{E}-04$ & $1.80 \mathrm{E}-04$ & $4.49 \mathrm{E}-05$ \\
$E_{N, \tau}$ & $1.18 \mathrm{E}-02$ & $0.29 \mathrm{E}-02$ & $7.18 \mathrm{E}-04$ & $1.80 \mathrm{E}-04$ & $4.49 \mathrm{E}-05$ \\
\hline
\end{tabular}

Table 8 Results for Example 5.4: convergence rates $r_{l}, N_{l}=32 \times 2^{l-1}, \quad l=1(1) 4$

\begin{tabular}{lllll}
\hline$\varepsilon$ & $r_{1}$ & $r_{2}$ & $r_{3}$ & $r_{4}$ \\
\hline $2^{-6}$ & 1.7843 & 1.9343 & 1.9844 & 1.996 \\
$2^{-8}$ & 1.7327 & 1.9156 & 1.9795 & 1.9954 \\
$2^{-10}$ & 1.1155 & 1.9069 & 1.9142 & 1.9762 \\
$2^{-16}$ & 2.0247 & 2.0134 & 1.9996 & 1.9993 \\
$2^{-18}$ & 2.0247 & 2.0134 & 1.9996 & 1.9993 \\
$2^{-20}$ & 2.0247 & 2.0134 & 1.9996 & 1.9993 \\
$\vdots$ & $\vdots$ & $\vdots$ & $\vdots$ & $\vdots$ \\
$2^{-50}$ & 2.0247 & 2.0134 & 1.9996 & 1.9993 \\
$R_{N, \tau}$ & 2.0247 & 2.0134 & 1.9996 & 1.9993 \\
\hline
\end{tabular}

Acknowledgments The research contained in this paper was supported by the National Research Foundation of South Africa. We thank the anonymous reviewers for their constructive comments. 


\section{References}

Clavero C, Jorge JC, Lisbona F, Shishkin GI (2000) An alternating direction scheme on a nonuniform mesh for reaction-diffusion parabolic problem. IMA J Numer Anal 20:263-280

Clavero C, Jorge JC, Lisbona F (2003) A uniformly convergent scheme on a nonuniform mesh for convection-diffusion parabolic problems. J Comput Appl Math 154:415-429

Clavero C, Gracia JL (2005) High order methods for elliptic and time dependent reactiondiffusion singularly perturbed problems. Appl Math Comput 168:1109-1127

Clavero C, Gracia JL (2010) On the uniform convergence of a finite difference scheme for time dependent singularly perturbed reaction-diffusion problems. Appl Math Comput 216:1478-1488

Doolan EP, Miller JJH, Schilders WHA (1980) Uniform numerical methods for problems with initial and boundary layers. Boole Press, Dublin

Shishkin GI (1986) Solution of a boundary value problem for an elliptic equation with a small parameter for the leading derivatives. USSR Comput Math Math Phys 26:38-46

Shishkin GI (2005) Robust novel high-order accurate numerical methods for singularly perturbed convection-diffusion problems. Math Model Anal 10(4):393-412

Roos H-G, Stynes M, Tobiska L (1996) Numerical methods for singularly perturbed differential equations. Springer, Berlin

Munyakazi JB, Patidar KC (2010a) Limitations of Richardson's extrapolation for a high order fitted mesh method for self-adjoint singularly perturbed problems. J Appl Math Comput 32:219-236

Munyakazi JB, Patidar KC (2010b) Higher order numerical methods for singularly perturbed elliptic problems. Neural Parallel Sci Comput 18:75-88

Munyakazi JB, Patidar KC (2012) Novel fitted operator finited difference methods for singularly perturbed elliptic convection-diffusion problems in two dimensions. J Differ Equ Appl 18(5):799-813

Jorge JC, Bujanda B (2004) Numerical methods for evolutionary reaction-diffusion problems with nonlinear reaction terms. J Comput Appl Math 166:167-180

Miller JJH, O'Riordan E, Shishkin GI (1996) Fitted numerical methods for singular perturbation problems. World Scientific, Singapore

Lubuma JM-S, Patidar KC (2006) Uniformly convergent non-standard finite difference methods for self-adjoint singular perturbation problems. J Comput Appl Math 191:228-238

Patidar KC (2005) High order fitted operator numerical method for self-adjoint singular perturbation problems. Appl Math Comput 171(1):547-566

Patidar KC (2007) High order parameter uniform numerical method for singular perturbation problems. Appl Math Comput 188:720-733

Hemker PW, Shishkin GI, Shiskina LP (2000) $\varepsilon$-uniform schemes with high-order time accuracy for parabolic singular perturbation problems. IMA J Numer Anal 20:99-121

Mickens RE (1994) Nonstandard finite difference models of differential equations. World Scientific, Singapore 
Rao SCS, Kumar S (2011) An almost fourth order uniformly convergent domain decomposition method for a coupled system of singularly perturbed reaction-diffusion equations. J Comput Appl Math 235:3342-3354

Linß T, Stynes M (1999) A hybrid difference scheme on a Shishkin mesh for linear convection-diffusion problems. Appl Numer Math 31:255-270

Linß T, Madden N (2010) Analysis of an alternating direction method applied to singularly perturbed reaction-diffusion problems. Int J Numer Anal Model 7(3):507-519 